\title{
Quaderni
}

QUADERNI Communication, technologies, pouvoir

\section{Représentations de la métropolisation : nouveaux lieux, nouveaux territoires}

\section{Bertrand Morvan}

\section{(2) OpenEdition}

Journals

\section{Édition électronique}

URL : http://journals.openedition.org/quaderni/772

DOI : 10.4000/quaderni.772

ISSN : 2105-2956

\section{Éditeur}

Les éditions de la Maison des sciences de l'Homme

\section{Édition imprimée}

Date de publication : 5 janvier 2014

Pagination : 101-112

\section{Référence électronique}

Bertrand Morvan, "Représentations de la métropolisation : nouveaux lieux, nouveaux territoires », Quaderni [En ligne], 83 | Hiver 2013-2014, mis en ligne le 01 janvier 2016, consulté le 19 avril 2019. URL : http://journals.openedition.org/quaderni/772 ; DOI : 10.4000/quaderni.772 


\section{Politique}

\section{Représentations de la métropolisation :} nouveaux lieux, nouveaux territoires

Bertrand Morvan

Conseil en développement territoirial
Les métropoles posent, comme objets d'étude et réalités spatiales et matérielles, un problème de définition pour tous les géographes ${ }^{1}$. « Métropole » et par incidence, «métropolisation » (soit le processus qui y conduit) sont donc des notions que l'on utilise sans qu'elles ne s'appuient aujourd'hui, sur des représentations globales et claires pour tout le monde. Pour le cas français, un praticien de l'aménagement urbain, J. Grangé, directeur de l'aménagement à Tremblay-enFrance, nous disait en juin 2013 : «Personne ne sait vraiment ce qu'est la métropole, et pourtant, on la fait quand même tous les jours! ». Cette remarque nous avait interloqué. Elle nous paraît faire l'intérêt d'un questionnement autour d'une telle position de praticien de l'urbanisme. Y-a-t-il une doctrine, une pensée de la métropole ou estil possible de "faire sans », comme le suggérait ce praticien? Nous tombions alors d'accord sur le fait d'enquêter sur les représentations de la métropole des praticiens in situ, afin de sortir d'une approche de type structurale, trop normative car imposée d'en haut par une géographie trop académique. Elle pouvait «passer à côté » des contextes urbains dans lesquels les acteurs se débattent pour construire la métropole. Nous assistions à un paradoxe : ceux qui développent la part conceptuelle, et donc normative d'une définition des formes métropolitaines, constataient que cette phase est difficile et inachevée. Nous nous intéresserons donc au rapport entre mots, représentations des acteurs et récit métropolitain (cela formerait un triangle dont les sommets interagissent du point de vue sociolinguistique). Une problématisation du sens caché de ce récit renverra aux composantes qui lui sont attachées. Cela nous amènera à aborder, dans une première partie, le problème de définition de la métropole 
et de la métropolisation. Nous identifierons comment les métaphores ("idées-images » selon Serge Moscovici en 1980) associées à des concepts prétendent organiser cette difficulté à l'aide d'un récit positiviste de la métropole. Notre hypothèse est qu'elles sont en partie cachées pour modeler l'image de chaque espace métropolitain, alors qu'il est « en phase toute récente d'expansion du processus urbain suscitant d'énormes mutations de style de vie... » et que, selon plutôt l'acception anglo-saxonne, cela se traduirait par la description suivante : «La qualité de vie urbaine, de même que la vie urbaine elle-même, est désormais une marchandise réservée aux plus fortunés, dans un monde où le consumérisme, le tourisme, les industries de la culture et de la connaissance sont devenus des aspects majeurs de l'économie politique urbaine $»^{2}$. Dans le cas français, nous pouvons émettre quelques doutes par rapport à une analyse aussi générale qui n'intègre pas suffisamment la position des catégories sociales moyennes dans la vie métropolitaine urbaine parisienne ${ }^{3}$. C'est donc bien l'analyse des composantes idéologiques d'un récit urbain de la métropole, avec sa fonction intégrative pour certaines catégories sociales « ciblées » qui nous intéresse ${ }^{4}$. Nous déboucherons, dans une deuxième partie, sur une lecture pragmatique du problème d'une métropolisation, faisant la part aux écarts et contradictions des projets urbains, lors de phases pratiques d'action.

\section{Métropole, métropolisation : un «bricolage» méthodologique pour compenser une difficulté de définition}

La difficulté à stabiliser les notions de métropole et de métropolisation, comme l'illustre l'exemple lorrain, nous empêche donc d'avoir une pensée unitaire de la métropole. L'acteur de la métropole, lui, va devoir proposer des solutions qui appellent, à chaque fois, un « mécano » ou une technologie de projet avec un ensemble d'intervenants. Cela explique l'utilisation du terme « bricolage». Alors que les contextes locaux d'intervention correspondraient à une nouvelle forme d'expansion du capitalisme dans l'espace, peut-on parler de « pensée de la métropole » ou du phénomène qui la structure avec la métropolisation ? Comment se représenter la métropolisation et quelles sont les représentations sur lesquelles l'édile pourra se fonder pour faire le moins d'erreurs possibles dans le processus décisionnel ? Ne s'agit-il pas, au-delà de toute technologie de la gouvernance, plutôt de la réalité d'une approche impressionniste qui se conjugue avec tous les modèles de la métropolisation? Nous nous demandons aussi s'il ne vaut pas mieux partir de cas pour « aller vers » une typologie caractérisant les formes de la métropolisation. On abandonnerait alors la question d'un idéal-type préalablement construit scientifiquement et il faudrait faire fond sur l'enquête empirique.

Des approches multiples et un cadre théorique incertain de la métropole

C'est une raison pratique qui force à interroger les représentations dont les acteurs des métropoles se servent pour concevoir la métropole et intervenir sur des formes ou des territoires en émergence. Comme l'explique V. Descombes (in Esprit n 3-4 de mars-avril 2011, Avancées et reculs démocratiques, entretien intitulé : «Comment agir en citoyen ?»), la raison pratique c'est celle de l'édile qui doit arbitrer et trancher en prenant 
le risque d'une décision qui « dessine l'avenir » dans un environnement de plus en plus incertain. Les délibérations préalables d'une éthique de la discussion avec toutes les expertises complémentaires ne «feront » pas la décision elle-même. C'est aussi la raison pratique de l'aménageururbaniste qui a pour mission de construire la forme urbaine en fonction d'un idéal de communauté d'appartenance.

Pourtant, on ne peut parler de métropolisation sans donner une définition générale de la métropole montrant le problème de la multiplicité de représentations des acteurs qui tentent de la définir pour agir sur ses territoires. Dans le dictionnaire de géographie dirigé par Y. Lévy et $\mathrm{P}$. Lussault de 1996, on trouve page 609 : « Métropole : espace urbain qui, tout en permettant la participation des acteurs aux processus d'échelle mondiale, reste une société locale ». S'y ajoute la définition d'une mégapole : "L'espace mégapolitain commence là où la configuration de l'espace urbain rend une gestion locale (liée au quotidien ou au familier) impossible ». Les auteurs soulignent que le constat de départ peut sembler décourageant et citent d'autres auteurs : «Il n'existe pas (...) de théorie unificatrice qui rende compte d'une manière satisfaisante des divers aspects du phénomène urbain » (D. Pumain et M.-C. Robic, 196, p.107) et ajoutent : «Le vocabulaire est si peu stabilisé, objet de débat et d'appropriations tellement contradictoires que l'on ne doit pas oublier que parler de métropole, c'est se référer à un modèle qui n'est pas encore arriver à maturité » (Lévy, 1995, p. 57). Depuis l'analyse des auteurs en 2001, il faut ajouter de nouveaux critères permettant de qualifier la métropole. Le développement des fonctions d'intermédiation et de conseil autour des activités financières concentrées en des lieux précis de celle-ci (nœuds de réseaux de communication, etc.) est à retenir (L. Halbert, 2012).

Une sémantique, associée à un constructivisme social, appelle à un questionnement sur la nature du récit métropolitain

Notre hypothèse est la suivante : les récits métropolitains ont pour finalité, par le jeu des institutions locales, la réunion d'acteurs autour de traditions d'action. Mais au départ, le récit s'inscrit dans un future proche au point de présenter une vision stratégique du développement local. Le récit est souvent en rupture avec le passé (industriel, etc...) avec son caractère futuriste (il donne un nouvel avenir au territoire), mais pas toujours. Si nous voulons faire la critique de ce récit, deux positions peuvent aider à se situer par rapport à l'incertitude dans la définition des notions-concepts comme « métropole » et « métropolisation », incertitude que l'on retrouve aussi bien dans le milieu de la recherche urbaine que dans celui des praticiens.

1) La position normative du dictionnaire, familière aux géographes. Il s'agit d'un savoir cumulatif, fait d'échanges en séminaires de chercheurs. Il illustre un travail d'actualisation des connaissances et de tri conceptuel inter-dictionnaires. La « praxis » des acteurs y intervient peu.

2) La position descriptive de l'usage des mots à travers l'histoire, les cultures et les contextes d'utilisation. Elle est permise par l'approche lexicale. C'est le projet d'un historien de la ville comme Christian Topalov dans son lexique ${ }^{5}$. 
L'hypothèse est la suivante : il faut s'ancrer dans les contextes d'utilisation des mots dont le sens a évolué du tout au tout parfois, dans l'histoire et selon les cultures d'origine. Le directeur de l'équipe dit, dans la préface, aux intervenants de ce futur lexique : «Ne jugez pas, mais citez des occurrences! ». Le mot n'est jamais neutre du fait des contextes historiques et socioculturels urbains où son usage est situé. L'intérêt de son projet est alors « centré » sur les changements de significations. Des variations de sens apparaissent avec les occurrences attachées à une même « entrée » lexicale sur un même espace national (l'évolution historique du sens d'un même mot est étonnante, selon l'auteur). Mais les sciences sociales britanniques ont contribué, tout récemment, à en étendre l'usage. Selon Topalov et alii : «A partir des années 70, les géographes tendirent à substituer metropolitan cities (villes) au terme plus ancien de great cities (grandes villes) pour désigner les villes qui se trouvent au sommet des systèmes hiérarchisés des lieux centraux (central places), y compris de sous-systèmes régionaux » (Robson, 1973).

Nous observons que l'idée de domination constitue un élément de continuité entre les usages contemporains et les usages plus anciens. La prédominance de Londres et l'usage de the Metropolis pour la désigner ont contribué de façon significative à déterminer le sens de metropolis et metropolitan en général, mais aussi l'usage actuel de ce dernier terme pour signifier un statut social impliquant un style et des goûts distingués, informés et cosmopolites. Cet élément de domination existe aussi dans l'extension du terme metropolitan des villes aux sociétés. «Dans l'usage actuel, les pays d'Europe occidentale et
d'Amérique du Nord sont souvent qualifiés de metropolitan, impliquant par là qu'ils sont les centres d'un pouvoir global » (David A. Reeder).

Cette deuxième position théorique nous paraît particulièrement intéressante pour permettre de considérer des écarts de sens qu'il faudra, ensuite, interpréter et traduire pour construire un point de vue normatif. On suspend ainsi une approche normative immédiate pour se donner le temps d'une compréhension in situ du phénomène métropolitain et du discours qui l'accompagne. Notre approche est plutôt tournée vers les représentations urbaines actuelles des acteurs de la métropole et moins vers l'histoire généalogique des mots de la ville. Ces représentations renvoient aux discours et aux représentations de référence (de formation au sens large et de contexte professionnel d'origine) des acteurs à interviewer.

Nous aurons à considérer la nature de la relation entre solutions locales envisagées et cadre théorique proposé. Dans quelle mesure peut-on parler de doctrine d'action et d'idéal-type de métropolisation? Y a-t-il un paradigme de la métropolisation, comme certains auteurs l'avancent (C. Ghorra-Gobin, 2010) ? Par ailleurs, notons que d'anciens théoriciens de la métropolisation et/ou de la métropole « passent au terrain » pour expérimenter opérationnellement leurs approches. Pourquoi ces mouvements, encore peu courants en recherche urbaine ? Cela répond-il à la nécessité d'une « cross-fertilisation » difficile entre lieux de la théorie et lieux de la pratique professionnelle avec la création d'espaces ad hoc de métissage des deux domaines ? F. Ascher, chercheur et organisateur de la recherche sur les métropoles, en parlait déjà se plaignant du manque 
de concepts adéquats et d'institutions adaptées pour le permettre (cf. pp. 26-27, son Manifeste pour la recherche urbaine et ses recommandations adressées aux institutions scientifiques et aux acteurs de la ville dans l'ouvrage intitulé Organiser la ville hypermoderne pour son Grand Prix de l'urbanisme de 2009)?

\section{Instable et complexe, la réalité exige des acteurs une inventivité narrative}

Du côté des praticiens, il faudrait identifier des figures de rhétorique privilégiées. Elles correspondraient à la légitimation d'un moment propice de l'action dans une localité (ex. : métaphores comme la machine, l'organisme de la ville et ses entrailles que sont les réseaux techniques urbains, le métabolisme urbain, le centre principal « commandant» les centralités périphériques des couronnes urbaines de la banlieue, la «polycentralité maillée » avec ses pétales de marguerite formant la région urbaine pour l'île-de-France, etc.). On peut partir ici, de ce qu'en dit Marcel Roncayolo dans le livre La ville et ses territoires au chapitre « Représentations et idéologies de la ville»p.176, au sous-chapitre « Représentations, images et pratiques de la ville ». Il montre que les aspects idéologiques sont présents dans les modes d'action sur la ville avec les courants « culturalistes » et «progressistes » qui ont vu l'avènement de l'urbanisme. Cela pourrait renvoyer à la définition de l'historienne de l'urbanisme F. Choay qui le définit de la façon suivante : «Vers la fin $d u$ $X I X^{e}$ siècle, l'expansion de la société industrielle donne naissance à une discipline qui se distingue des arts urbains antérieurs par son caractère réflexif et critique et par sa prétention scientifique» (F. Choay, 1965). Pour le géographe P. Merlin :
"Cette définition situe bien les travaux des théoriciens qui ont prétendu fonder l'urbanisme. Elle correspond moins bien à un champ professionnel qui a rapidement pris une dimension concrète et focalise des enjeux (y compris financiers) importants » (dans le Dictionnaire de l'urbanisme et de l'aménagement, Paris, P.U.F., 2008). Le Grand dictionnaire encyclopédique Larousse (1982-1985) définit l'urbanisme comme : «L'art d'aménager et d'organiser les agglomérations humaines et de façon précise » ou encore «l'art de disposer l'espace urbain ou rural au sens large (bâtiments d'habitation, de travail, de loisirs, réseaux de circulation et d'échanges) pour obtenir son meilleur fonctionnement et améliorer les rapports sociaux » (dans le Dictionnaire de l'urbanisme et de l'aménagement, Paris, P.U.F., 1988, 4e éd., 2005, 2008). Il s'agit donc d'une « praxis scientifique » née pour accompagner l'industrialisation de la ville à l'aide de la modernité technique. C'est encore plus vrai quand l'outil technique (numérique souvent) est brandi pour prétendre à une réalisation idéale accompagnée d'une évidente «neutralité » du point de vue des acteurs. Par exemple (cf. l'intervention de F. Grosse, directeur technique de Veolia Environnement intitulée : «Quels enjeux pour les services urbains ? » au dernier colloque sur le thème Faire la métropole par ses réseaux du 11 et 12 avril 2013, Institut d'Urbanisme de Paris), les présentations en « $3 \mathrm{D}$ » donnent l'apparence d'une forme d'objectivité dans la peinture de la situation à l'aide d'une carte des futurs réseaux techniques d'énergie intégrés par des silos sur certaines portions du territoire de l'agglomération. Cela est présenté comme souffrant peu la contradiction, particulièrement lorsqu'il s'agit de l'aménagement d'une grande agglomération, 
« Le Grand Lyon ». Pourtant, après avoir assisté à une de ces représentations, c'est le mot de complexité au sens d'E. Morin (E. Morin, 1986, 1990) qui ressort. Il s'agit de multiples relations qu'on nous donne à voir avec des représentations graphiques de flux pratiquement en fonction sur tout l'ensemble du Grand Lyon. Des éléments entre ces relations de flux sont peu définis (« les silos» qui sont autant de plateformes de connexion), ,comme nous l'apprend la théorie des systèmes qui fait appel à ce type de représentation. Mais la complexité non développée est illustrée par un récit de la future réalité construite sans nous donner un sens de la totalité avec des aspects contradictoires à la vision d'ensemble proposée. Le concepteur renvoie à la phase de négociation future du sens général du schéma dans sa totalité par les élus locaux et les ingénieurs concepteurs de la compagnie.

Des visions politiques plus ou moins abouties avec les récits métropolitains proposés.

Dans le cas brésilien de la construction métropolitaine, on voit que la forme se conçoit accompagnée du récit qui la légitime, avec l'effraction intempestive de la classe moyenne issue de la « société civile » qui le remettra en cause. Les dirigeants brésiliens ne s'y attendaient pas. Cet exemple pose, avec la notion de « capital urbain » soulevée par Sylvain Kahn, l'interrogation en amont suivante : «Le capital doit-il être utilisé pour financer des infrastructures sportives ou pour la création de biens publics ou services comme des infrastructures de transport? Les citadins sont-ils d'abord des spectateurs de spectacles de masse ou bien sont-ils d'abord des personnes libres de se déplacer grâce à l'aide de réseaux accessibles au plus grand nombre?". Pour le géographe : "Ce débat est né à Séoul avec lesJeux olympiques de 1988. Il s'est nourri à Barcelone, à Athènes, à Pékin. Il s'est nourri à l'Exposition universelle de Shanghai. Il s'est développé à la coupe du monde en Afrique du Sud et maintenant au Brésil ». Par rapport à la situation actuelle, il y aurait une différence : «De Séoul à Londres via la Cap et contrairement à ce qui se passe en ce moment, on a eu les jeux et le métro, à l'exemple du pain et des jeux d'une certine époque. Tandis qu'aujourd'hui, à Sao Paulo et Rio, que demande la jeunesse ? Elle dit : pas de jeux sans métro! C'est la grande nouveauté entre les grandes villes précédentes et Rio. Dans les villes organisatrices précédentes à Séoul, Athènes et même Barcelone en 1992, les manifestations sportives de niveau planétaire $s$ 'étaient traduites par des améliorations notables des infrastructures de transports, de communication et d'adduction. Et c'est là l'astuce, car en dépit de la croissance du mètre carré, ce bénéfice avait permis l'assentiment des classes moyennes émergentes. Or il y a quelque chose qu'on n'a pas forcément vu à ces occasions dans le contrat moral - je vous échange des grands stades contre des infrastructures urbaines. Ces évènements avaient fait l'objet d'un grand récit hollywoodien merveilleux : celui de mégapoles modernes, agréables, attractives, de villes offrant tous les avantages du capital urbain sans offrir les inconvénients du gigantisme et du mal développement ». Ce « récit mégapolitain » aurait une autre fonction, mais celle-ci cachée : "Il s'agit de contenir, de cacher dans une invisibilité relative les habitants des bidonvilles détruits et des quartiers transformés par ces chantiers urbains. Car chassés par ces gargantuesques et juteuses 
opérations immobilières, ces «perdants » pauvres des J.O. et des Mondiaux de football se révoltent peu. Quand ils le font, ils sont facilement mâtés. Ils sont faiblement indemnisés. Ils sont relogés et relégués à 2 ou 3 dizaines de kilomètres de chez eux ». D'après S. Kahn : "Si l'on prend le cas de Rio, personne n'en parle encore. Mais il $y$ aurait bien 160000 cariocas dans ce cas. Aux J.O. de 2008, il y a eu 400000 Pékinois délogés par le pouvoir "à la baguette ». Il en détient la médaille d'or. Mais à Londres en 2012, il y a eu une véritable « gentrification» du quartier populaire de Stratford. Londres mérite ainsi la palme de l'« embourgeoisement» le plus réussi ». Et il ajoute : «À ce Monopoly planétaire se joint le plus beau récit urbain et pour le dire avec les mots du rugby, les dirigeants des villes et du Brésil, confrontés à ces classes moyennes, décrochent la cuillère de bois ».

On peut penser aussi aux machines de l'île de Nantes qui sont l'expérience même, depuis 2007 , d'une vision stratégique de reconquête urbaine métropolitaine d'une partie de Nantes avec les coauteurs François Delarozière et Pierre Orefice : « Projet artistique totalement inédit, il naît de l'imagination des deux auteurs à la croisée des « mondes inventés » de Jules Verne, de l'univers mécanique de Léonard de Vinci et de l'histoire industrielle de Nantes, sur le site exceptionnel des anciens chantiers navals $»^{6}$. Il s'agit de comprendre que derrière toute avancée sur la « métropole créative » d'un Richard Florida ${ }^{7}$, il $\mathrm{y}$ a des contradictions spatiales et culturelles à l'endroit où des quartiers en difficulté regroupent les « laissés pour compte de la métropolisation». Il faudra donc s'intéresser, lors de la critique du récit métropolitain, aux éléments multiples et éclatés, sous-jacents à un récit linéaire. Ce récit est nécessaire à la création d'une nouvelle histoire urbaine. Elle a pu ne pas retenir certaines parties de la mémoire ouvrière et en conserver d'autres, comme pourrait le montrer l'exemple de l'Île de Nantes. Il existe alors toujours des « points aveugles » du « storytelling » (C. Salmon, 2007) tel qu'il est présenté par l'édile et ses communicants. Ce récit complexe privilégiera toujours les " cibles » économiques et culturelles que sont les classes moyennes supérieures (intégrant les artistes) auxquelles il s'adresse.

Les caractéristiques du phénomène métropolitain pourront être appréhendées dans les contextes locaux d'intervention en confrontant les différentes représentations données pour le qualifier. Les géographes retiennent la définition de Jean-Paul Guérin : "Création sociale et/ou individuelle d'un schéma pertinent du réel » (J.P. Guérin, H. Gumuchian, I.G.A., 1985). Pour Denise Jodelet, la définition d'une représentation sociale est : « (...) une forme de connaissance, socialement élaborée et partagée, ayant une visée pratique et concourant à la construction d'une réalité commune à un ensemble social » (D. Jodelet, 1993, p. 36). Les catégories nous paraissent peu distinguées alors que les acteurs cherchent à « aménager la société » en « créant l'avenir en plan » (J.P. Gaudin, 1979, 1987, 1995). En fait, le chercheur s'attachera à décrypter les images (« idées-images » pour S. Moscovici, 1981) que les individus et les institutions se font de la réalité et celles qui lui sont proposées. Il s'intéressera, par exemple, au sens commun qui s'en dégage à la suite d'actes de préfiguration d'un projet urbain des architectes-urbanistes à l'aide de supports visuels (projections iconographiques, 
en 3D, plans, etc.). L'approche d'un objet par la géopolitique doit passer «systématiquement (par) une critique des représentations, en les confrontant les unes aux autres, pour saisir leurs contradictions » (Dictionnaire de géopolitique, 1993). La critique s'étendra autant à l'objet qu'au chercheur lui-même. Il faut alors pour aborder un objet, le déconstruire et analyser chacune des images (et donc usages des mots) que se font de lui les différents acteurs.

Des images symboliques et des métaphores nécessaires à la vision de l'organisation future de l'espace métropolitain

Si nous identifions toutes les figures-images qui incarnent les mots métropole et métropolisation dans le discours des acteurs-experts, la réalité apparaît comme un ensemble aux contours flous. L'hétérogénéité des situations ne permet que difficilement de dégager des caractéristiques aboutissant à un idéal-type de ce qu'est la métropolisation. Si l'on peut visualiser des formes urbaines et les représentations de l'espace émergentes avec par exemple, un étalement urbain et une concentration des sites de commandement économique sur des «points», une pléthore de métaphores doivent être interrogées. Considérer leur efficacité symbolique comme des visions stratégiques du développement urbain à destination des acteurs devient essentiel. Ainsi nous nous demanderons à la suite : ce qu'est une représentation qui a pour modalité l'image (S. Moscovici, 1980, J.P. Guérin, Y. Lacoste...), ce qu'est une « vision stratégique » qui « prend» ou « ne prend pas » par rapport à l'incertitude pressentie de l'avenir qu'on cherche malgré tout à définir ( « futuribles »; schémas prospectifs avec des scénarios...).

Prenons l'exemple du concept de «métropole « post- suburb» ou métropole de l'aprèsréseaux » de O. Coutard, S. Weglenski et G. Dupuy développé au colloque intitulé Faire la métropole par ses réseaux du 11 et 12 avril de l'Institut d'Urbanisme de Paris. Nous notons comment les experts-modélisateurs vont être inspirés par l'idée de rattraper ce qu'ils qualifient d'avancées des pratiques de mobilité métropolitaine. Ces dernières sont appréhendées, parfois de manière qui nous paraît discutable, comme en avance sur les modèles. Pour O. Coutard : «Le fait que les réseaux techniques, structurant au niveau macro-géographique de la région urbaine, ne résolvent pas les controverses et équivoques que pose la question de la construction de la territorialité en banlieue (cf. dans leur dernier livre l'analyse commune de M. Wiel et J.P. Orfeuil concernant l'urbanisation densifiée des gares dans le cadre du projet de métro automatique du Grand Paris). C'est le problème de l'interconnexion généralisée plutôt que la «fin de l'urbain»». Pour G. Dupuy, la métropole de l'après-réseaux ou « post-suburb » peut être définie de la façon suivante : «Il s'agit d'une dynamique, d'un processus de différenciation interne des espaces de banlieue opposé à la nappe suburbaine. Il y a recréation de centralités selon les points $d u$ territoire avec des communes «qui gagnent » et des communes «qui perdent»».

On voit bien ici que dans le cadre de la signature des 19 contrats de développement territoriaux avec l'État pour la réalisation des objectifs métropolitains en 2012, l'avenir de villes de 20000 habitants en périphérie de Paris et hors des 
secteurs de développement des «pétales de la marguerite » va être une question posée. P. Mansat, adjoint au maire de Paris B. Delanoë, les qualifiera de « territoires spécifiques à traiter » (Assises de 1'A.M.I.F. de la ville nouvelle des 9-10-11 avril 2013 à Vincennes, Atelier « Grand Paris : où allons-nous? »). Tout se négociera au niveau local, avec des groupes d'acteurs à géométrie variable et dans le secret de conciliabules entre édiles autour de projets attracteurs (exemple : le centre du cancer à Villejuif qui réunit un ensemble de communes autour de la communauté d'agglomération du Val de Bièvre et son vice-président M. Le Bouillonnec, responsable du développement social urbain et députémaire de Cachan).

Des problèmes sont apparus avec le développement des réseaux dans de nombreuses activités urbaines (eau, assainissement, automobile, transports, etc...). Cet accroissement a produit l'étalement urbain chronique, des menaces de fractures territoriales et de dépendance aux macrosystèmes techniques (constat général de Marvin et Graham en 2001 pour les T.I.C, énergie et T.C.). Selon O. Coutard, la connexion est de plus en plus nécessaire au point que cela se généralise avec l'équipement en voieries et voies diverses (V.R.D.) des communes et les réseaux électriques. Cela pose aussi, la question des enjeux liés aux problèmes de financement de ces infrastructures. La course au développement des réseaux techniques lourds tourne à l'impasse politique. Pour G. Dupuy : «La solidité économique du développement des réseaux ne compense pas les fragilités sociales et économiques de cette tendance à la croissance exponentielle ». Or le projet du Grand Paris Express va vers cette tendance « sans avoir la variation de planification dans les projets et la souplesse des premiers théoriciens (Cerdà et Soria y Mata). La solution de contrôle du système territorial fou serait du côté des « Smart grids», des T.I.C. et du transport $»$. Il faudrait observer les pratiques de mobilité et de consommation, avant de « figer les réseaux dans le marbre » en ce qui concerne le projet du Grand Paris.

Une autre « idée-image » est le « localisme métabolique » (O. Coutard, J.-M.Offner, D. Béhar...). C'est une représentation à caractère conceptuel qui se traduit de la façon suivante : " Il s'agit de l'intégration globale des grands réseaux à l'échelle de la région urbaine. En fait cela dénote une complémentarité plutôt qu'une opposition entre échelle globale et échelle locale avec le niveau intermédiaire. On parle de « métabolisme environnemental » pour la logique d'intégration à grande échelle et pas réellement de tension avec «le retour du local»». Ce sont des représentations qui vont inspirer l'intervention des praticiens. La critique de l'action des réseaux techniques, historiquement présents pour faire la métropole, montre comment on passe d'une phase de promotion de l'action des réseaux à une phase de critique avec le passage à un seuil de contreproductivité (I. Illitch, 1980) du développement de la « rétistique » (G. Dupuy, 1991). Elle prend alors le pas d'une part, sur les nouveaux objectifs du développement durable et d'autre part, elle pose le problème de la dépendance vis-à-vis des macro-systèmes techniques (A. Gras, 1997).

Cette phase d'analyse des différents auteurs, tous inspirés par le paradigme de l'urbanisme des réseaux, nous invite à l'interpréter comme une phase descriptive de raisonnement. L'avenir des 
villes de la périphérie de banlieue métropolitaine est interrogé après la phase que nous qualifions de « normative ». Pourtant, il faut noter une difficulté à appréhender les différentes singularités qui font le phénomène de la métropolisation, difficulté dont témoigne le dernier ouvrage intitulé Suburbia d'un auteur phénoménologue comme B. Bégout (2013) ou encore Y. Chalas et G. Dubois-Taine avec « la ville émergente » (1995).

\section{Conclusion}

Nous pouvons conclure sur le caractère relatif d'un modèle et d'une pensée de la métropole. Il faut pourtant nuancer cette considération, dans la mesure où il paraît nécessaire, si l'on suit le géographe marxiste D. Harvey après H. Lefebvre, de réintégrer les aspects déterministes des lois du capital dans la phase d'expansion économique mondialisée à laquelle nous assistons. Il s'agit de grosses opérations de renouvellement urbain des centres-villes et d'extension des grandes métropoles, capitales financières du monde et nœuds essentiels de communication.

Ce que nous qualifions de « pensée de la métropole », avant d'investiguer d'autres métropoles françaises dans l'avenir, dépendrait essentiellement, de la nature de la construction d'une représentation collective des acteurs. Cette dernière peut s'appréhender comme le produit d'une technologie d'ajustement entre acteurs et un " work in progress » au titre de projet urbain (G. Pinson, 2005), dans un contexte donné géographique et historique. Il faudra donc toujours questionner les contradictions de la production de l'espace qui s'en dégagent, par-delà les représentations de l'espace des décideurs d'un renouvellement urbain. C'est le cas du projet du Grand Paris avec 1'histoire de l'opposition entre Paris et sa banlieue.

Pour l'analyste d'une « praxis » des urbanistes, il serait souhaitable de rester dans une forme de systématique ouverte ${ }^{8}$, non-dogmatique, face au phénomène mondialisé d'une technique et d'un urbanisme postmodernes. Interroger la forme métropolitaine supposerait donc de s'appuyer sur la raison pratique des acteurs qui ne théorisent pas nécessairement lors du développement de leurs projets. Cela exige d'une part de s'appuyer sur la vie quotidienne des urbains qu'il faut décrire, et d'autre part d'analyser in situ les formes de légitimation politique des décisions à l'aide des représentions des acteurs de la ville. Modes de décisions fortement liés à d'importants enjeux financiers et aux contextes politiques sousjacents.

De fait, nous avons affaire à de nouvelles formes urbaines où les dimensions culturelles de nouveaux styles de vie fortement suggérées par les décideurs - soit les récits métropolitains et les figures symboliques qu'ils font naître - jouent une partition essentielle. Ces formes devront être appréhendées à partir de l'histoire des usages des mots des acteurs de la ville et la théorisation qui en est faite souvent après coup. Toute leur singularité lexicale devra être intégrée comme le ferait un bon traducteur, en faisant place aux différences culturelles nationales et à l'évolution historique des significations que prennent les mots avec leur usage. 


\section{$R \cdot E ́ E \cdot E \cdot E \cdot R \cdot E \cdot N \cdot C \cdot E \cdot S$}

P.L. BERGER, T. LUCKMAN, The social construction of reality, 1966, Armand Colin, Paris, 2006.

G. DUPUY, L'urbanisme des réseaux - Théories et méthodes, Armand Colin, Paris, 1991.

M. RONCAYOLO, La ville et ses territoires, Gallimard, Folio-Essais, Paris, 1990.

D. HARVEY, Le capitalisme contre le droit à la ville - Néolibéralisme, urbanisation, résistances, Éditions Amsterdam, 2011.

D. PUMAIN, J.M. OFFNER (dir.) et alii., Réseaux territoriaux - Significations croisées, Éditions de l'Aube, 1996.

H. LEFEBVRE, Le Droit à la ville, Paris, Seuil, 1967, (réédition) 1970.

H. LEFEVBRE, L'idéologie structuraliste, Paris, Points-Seuil, 1971.

H. LEFEVRE, Production de l'espace, Paris, Anthropos, 1974.

C. GHORRA-GOBIN (dir.), Dictionnaire de la mondialisation, Armand Colin, Paris, 2013.

C. TOPALOV, L. COUDROY DE LILLE, J.C. DEPAULE, B. MARIN (sous la direction de), L'aventure des mots de la ville à travers le temps, les langues, les sociétés, Laffont Robert, Bouquins, Paris, 2010.

P. RICEUR, Idéologie et utopie, 1975, Seuil (réédition), Paris, 1997.

C. SALMON, Storytelling, la machine à créer des histoires et à formater les esprits, La Découverte, Paris, 2007.

H. BEVORT H., A. ROUSSEAU A. : « La banlieue, mythe politique français » (pp. 83-97), Esprit $\mathrm{n}^{\circ} 393$, Tous périurbains !, Paris, marsavril 2013.
B. BEgOUT, Suburbia. Autour des villes, Éditions Allia - Incultes, Bordeaux, 2013.

L. SFEZ, La politique symbolique, Quadrige, Paris, P.U.F., 1993, réédité en 1996.

P. LASCOUMES, P. LE GALÈS (dir.), Gouverner par les instruments, Paris, P.N.F.S.P., 2005.

L. HALBERT, L'avantage métropolitain, Paris, P.U.F., 2010.

E. MORIN, La connaissance de la connaissance (T.3), Paris, Points- Seuil, 1986, 1992 et Introduction à la pensée complexe, Paris, Seuil, 1990. S. SASSEN, The Global City. New York London, Tokyo, Princeton University Press, 1991.

LE DÉBAT $\mathrm{n}^{\circ} 80$, La ville globale. Éléments pour une lecture de Paris, p.146-164.

ESPRIT $\mathrm{n}^{\circ}$ 3-4, Avancées et reculs démocratiques, Paris, Mars-Avril 2011.

QUADERNI $\mathrm{n}^{\circ}$ 73, La métropole parisienne. Entre récits, paroles et échanges, Paris, 2010. Grand Prix de l'urbanisme de F. Ascher, Organiser la ville hypermoderne, Éditions Parenthèses, 2009. 


\section{$\mathrm{N} \cdot \mathrm{O} \cdot \mathrm{T} \cdot \mathrm{E} \cdot \mathrm{S} \mathrm{R} \cdot \mathrm{E} \cdot \mathrm{S} \cdot \mathrm{U} \cdot \mathrm{M} \cdot \mathrm{E}$}

1. Moriset (B.) : « La délimitation des aires métropolitaines - Représentations et enjeu politique - L'exemple de la métropole lorraine Nancy-Metz », Annales de géographie $\mathrm{n}^{\circ} 595,1997$, pp. 243-262). Pour l'auteur, il y a donc bien une "dilution » de la réalité géographique de la grande ville, qui devient un champ de forces sans limites précises comme le montre cet espace géographique.

2. Harvey (D.), Le capitalisme contre le droit à la ville - Néolibéralisme, urbanisation, résistances, p. 21, Paris, Éditions Amsterdam, 2011.

3. Preteceille (E.) : « Division sociale et globalisation. Le cas de la métropole parisienne » in Sociétés contemporaines $\mathrm{n}^{\circ} 22 / 23$, Éditions l'Harmattan, juin/ septembre 1995. Conférer l'analyse critique du sociologue-économiste E. Preteceille. Il prend distance par rapport à la vision de $\mathrm{S}$. Sassen au sujet de la division sociale de l'espace parisien. En particulier, il met en cause sa vision « en sablier » de la globalisation des villes avec sa dualisation sociale due à la croissance des catégories du tertiaire supérieur associée au déclin du groupe ouvrier dans son livre intitulé Global City (1991).

4. Ricœur (P.), Idéologie et utopie, Seuil, Paris, 1975, 1997.

5. Topalov (C.), Coudroy de Lille (L.), Depaule (J.C.), Marin (L.) directeurs, L'aventure des mots de la ville À travers le temps, les langues, les sociétés, Éditions Robert Lafond - Collection Bouquins, Paris, 2010.

6. Fascicule de présentation : «Bienvenue aux machines de l'île de Nantes », Nantes, 2013.

7. Florida (R.), Cities and the creative class, 2005 et The rise of the Creative Class, Routledge, 2002.

8. Axelos (K.), Systématique ouverte, Éditions de Minuit, Paris, 1984.
Mots et représentations des acteurs fondent le récit métropolitain. L'exemple de récit formulé par le Brésil avant la crise sociale de l'été 2013 est applicable à Bordeaux, Lyon, Marseille, Nantes et Paris, pour concevoir une typologie des récits « à la française». Une rhétorique a émergé chez des analystes de l'urbain métropolitain, qui peinaient à définir des métropoles en évolution. Un futur programme de recherche avec enquêtes de terrain devra recueillir les représentations de la métropole qu'emploient les praticiens de l'urbain, face aux nouveaux lieux et territoires sur lesquels ils interviennent. Nous faisons l'hypothèse d'un décalage entre théoriciens et praticiens, ceux-ci devant s'ajuster aux pratiques sociales dans l'espace, et à la demande politique. Dans le cas du Grand Paris, ce projet s'est formulé contre des représentations produites par l'opposition historique et mythologique : Paris contre banlieue. 